\title{
Sensor Node Localization Methods based on Local Observations of Distributed Natural Phenomena
}

\author{
Felix Sawo, Thomas C. Henderson, Christopher Sikorski, and Uwe D. Hanebeck
}

\begin{abstract}
This paper addresses the model-based localization of sensor networks based on local observations of a distributed phenomenon. For the localization process, we propose the rigorous exploitation of strong mathematical models of distributed phenomena. By unobtrusively exploiting background phenomena, the individual sensor nodes can be localized by only observing its local surrounding without the necessity of heavy infrastructure. In this paper, we introduce two novel approaches: (a) the polynomial system localization method (PSL-method) and (b) the simultaneous reconstruction and localization method (SRL-method). The first approach (PSLmethod) is based on restating the mathematical model of the distributed phenomenon in terms of a polynomial system. These equations depend on both the state of the phenomenon and the node locations. Solving the system of polynomials for each individual sensor node directly leads to the desired locations. The second approach (SRL-method) basically regards the localization problem as a simultaneous state and parameter estimation problem with the node locations as parameters. By this means, the distributed phenomenon is reconstructed and the individual nodes are localized in a simultaneous fashion. In addition, within this framework the uncertainties in the mathematical model and the measurements are considered. The performance of the two different localization approaches is demonstrated by means of simulation results.
\end{abstract}

\section{INTRODUCTION}

Recent developments in various areas dealing with sensor networks and the further miniaturization of individual nodes make it possible to apply wireless sensor network for observing natural large-area physical phenomena [1]. Examples for such physical quantities are temperature distribution [2], chemical concentration [3], fluid flow, structural deflection or vibration in buildings, or the surface motion of a beating heart in minimally invasive surgery [4].

For the reconstruction of such distributed phenomena, the individual sensor nodes are densely deployed either inside the phenomenon or close to it. Then, by distributing local information to a global processing node, the phenomenon can be coöperatively reconstructed in an intelligent and autonomous manner [5], [6], [7]. In such scenarios, the sensor network can be exploited as a huge information field collecting data from its surrounding and then providing useful information both to mobile agents and to humans. Hence, respective tasks are accomplished more efficiently, thanks to the extended perception provided by the sensor network. By this means, sensor networks can forecast or

F. Sawo and U. D. Hanebeck are with the Intelligent Sensor-ActuatorSystems Laboratory (ISAS), Institute of Computer Science and Engineering, University of Karlsruhe, Germany, sawo@ira.uka.de, uwe. hanebeck@ieee. org

T. Henderson and C. Sikorski are with the School of Computing, University of Utah, USA, tchecs.utah.edu, sikorskiecs.utah.edu

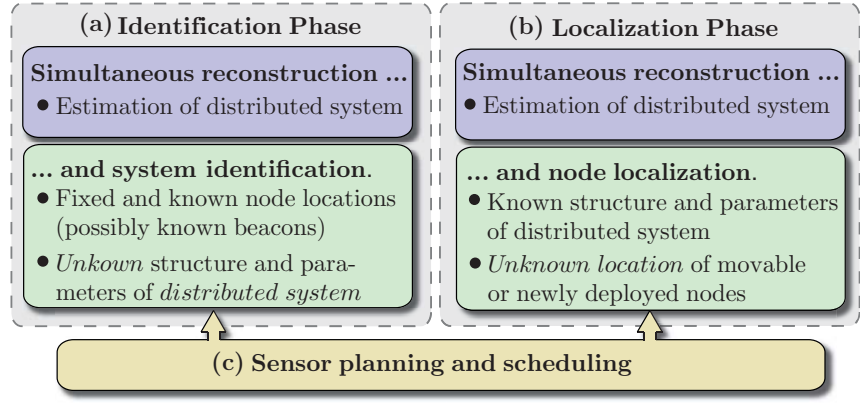

Fig. 1. Visualization of two possible phases for the estimation of distributed natural phenomena. The phases are managed by a planing and scheduling process (not considered in this paper). (a) The first phase consists of the identification of the environment in order to derive a strong mathematical model of the phenomenon to be monitored (identification phase) [6] (b) Based on the mathematical model, newly deployed sensor nodes or movable nodes can be localized by local observations (localization phase)

prevent dangerous situations, such as forest fires, seismic sea waves, or avalanches [8].

For most sensor network applications, the sensory data has only limited utility without location information. In particular for the accurate reconstruction of distributed phenomena, the locations of the individual sensor nodes are necessary. Manually measuring the location of every node in the network becomes infeasible, especially when the number of sensor nodes is large, the nodes are inaccessible or in the case of mobile sensor deployments. That makes the localization problem one of the most important issues to be considered in the area of sensor networks.

In general, localization methods could be classified into active methods and passive methods. The active localization methods estimate the locations based on signals that are artificially stimulated and measured by the sensor network, e.g., artificially generated acoustic events. That means, the localization is performed in controlled environments and incurs significant installation and maintenance costs. A comprehensive survey on active localization methods can be found in [9]. On the other hand, the passive localization methods occur in a non-controlled environment, where stimuli are generated in a natural and autonomous fashion. The advantage of passive methods is that they do not need additional infrastructure, and thus keep the installation and maintenance costs at a very low level. Furthermore, these methods become particularly important for applications, where satelite positioning systems are simply not available, e.g., indoor applications, sensor networks for monitoring the soil or snow cover. In our previous research work, a purely datadriven modelling approach was introduced for the passive 
localization of cellular phones based on measuring signal strengths [10] and barometric pressure [11].

For the passive localization of sensor nodes, we present model-based approaches based on local observations. The novelty of the methods introduced in this paper is the rigorous exploitation of a strong mathematical model of the distributed phenomenon for localizing individual sensor nodes. Furthermore, within this framework, the often remaining uncertainties in the sensor node locations can be considered during the reconstruction process of the distributed phenomenon [2]. The use of such a mathematical model for node localizations was proposed in [8]. However, there was no consideration of uncertainties naturally occuring in the measurements and in the used model.

In this paper, we introduce two different methods for the model-based passive localization of sensor nodes based on local observations: (a) the polynomial system localization method (PSL-method), and (b) the simultaneous reconstruction and localization method (SRL-method). The first approach (PSL-method) is purely deterministic, meaning that neither uncertainties in the model description nor in the measurements are considered. This direct method is based on restating the model of the distributed phenomenon in terms of a polynomial system including the state of the phenomenon and the location to be identified. Then, solving a system of polynomial equations leads directly to the desired location of the sensor node. The second approach (SRLmethod) considers uncertainties both in the mathematical model and the measurements during the localization process. It is shown that the localization problem can be regarded as a simultaneous state and parameter estimation problem, with node locations as the parameters to be identified. This leads to a high-dimensional nonlinear estimation problem, making the employment of special kinds of estimators necessary. By this means, the sensor nodes are localized and the distributed phenomenon is reconstructed in a simultaneous fashion. The improved knowledge can be exploited for other nodes to localize themselves.

The rest of the paper is organized as follows: Section II contains a rigorous formulation of the problem and challenges for localizing sensor nodes based on local observations of a distributed phenomenon. Section III describes a deterministic approach based on solving a polynomial system of equation (PSL-method). Section IV is devoted to spatial and temporal decomposition methods allowing the conversion of the distributed phenomenon into a system description in state-space form. Based on this system description, we derive a method for the simultaneous reconstruction of distributed phenomena and node localization (SRLmethod) in Section V. It turns out that this leads to a highdimensional nonlinear estimation problem, however, with a linear substructure. Accordingly, a novel estimator, the socalled Sliced Gaussian Mixture Filter [12], is employed. This allows exploitation of the (conditionally) linear substructure in the high-dimensional nonlinear estimation problem, and leads to a more efficient localization process.

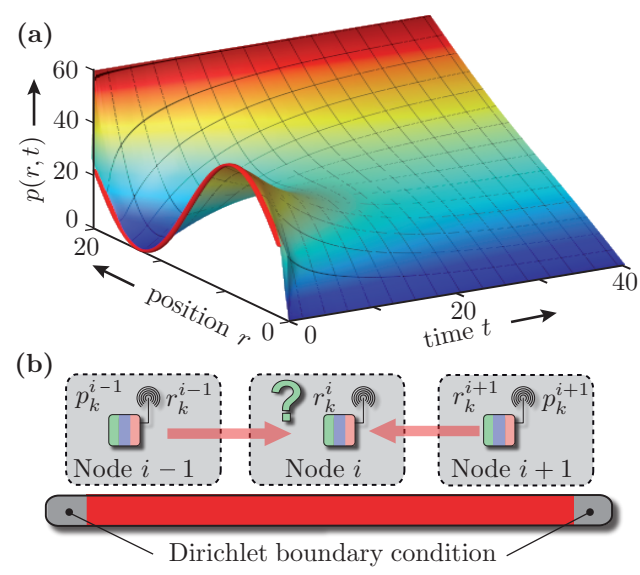

Fig. 2. (a) Numeric solution of the considered distributed phenomenon subject to Dirichlet boundary condition at both ends. (b) Simulation setup for localization based on a system of polynomial equations (PSL-method). The sensor node to be localized receives measurements of the distributed phenomenon and locations from neighboring nodes.

\section{Problem Formulation}

The main goal is to design a novel localization method for sensor network applications, where individual nodes are able to locally measure a distributed phenomenon only. We assume to have a strong mathematical model of the phenomenon, i.e., with known model structure and model parameters. This model could possibly result from an earlier identification phase, visualized in Fig. 1 (a). Based on this mathematical model and local measurements newly deployed or movable sensor nodes can be efficiently localized, without using a global positioning system, see Fig. 1 (b).

Throughout this paper, we consider the localization based on the observation of a distributed phenomenon described by the one-dimensional diffusion equation

$$
\mathbb{L}(\boldsymbol{p}(r, t))=\frac{\partial \boldsymbol{p}(r, t)}{\partial t}-\alpha(r, t) \frac{\partial^{2} \boldsymbol{p}(r, t)}{\partial r^{2}}-\boldsymbol{s}(r, t)=0,
$$

where $\boldsymbol{p}(r, t)$ denotes the distributed state of the phenomenon. The diffusion coefficient $\alpha(r, t)$ could be varying in both time and space. Given an estimated solution $\boldsymbol{p}(r, t)$, the aim is the estimation of the sensor node location $\boldsymbol{\eta}_{k}^{S}$ based on local measurements of a realization of the distributed phenomenon $\boldsymbol{p}(r, t)$. In this paper, we consider the worst-case scenario where the node location $\boldsymbol{\eta}_{k}^{S}$ is completely unknown and the phenomenon $\boldsymbol{p}(r, t)$ still contains some uncertainties; see Fig. 2. The same methods can be utilized for the purpose of simply considering uncertainties in node locations during the reconstruction of distributed phenomena.

First, we derive the deterministic approach (PSL-method), which needs less computational performance and can be implemented in a fairly straightforward manner. However it does not consider uncertainties at all. Then, the more involved localization method (SRL-method) is introduced, considering the uncertainties both in the mathematical model and in the measurements. In addition, it gives a measure for the uncertainty of the estimated location $\boldsymbol{\eta}_{k}^{S}$. 


\section{Polynomial System Localization Method}

This section is devoted to a deterministic approach for the localization of individual nodes in a sensor network based on local measurements of a distributed phenomenon. The key idea of the proposed direct method is to solve the partial differential equation (1) in terms of the unknown node locations. This leads to a straightforward solution as long as the resulting nonlinear equations can be readily solved. Solving these equations for all sensor locations is called the Polynomial System Localization Method (PSL-method). The PSL-method basically consists of two steps: (1) spatial and temporal discretization of the mathematical model, and (2) reformulating and finally solving the resulting system of polynomial equations in terms of the desired locations.

1) Spatial and Temporal Discretization: The simplest method for the spatial and temporal discretization of distributed phenomena is the finite-difference method [7], [8]. In order to solve the partial differential equation (1), the derivatives need to be approximated with finite differences according to

$$
\frac{\partial p(r, t)}{\partial t}=\frac{p_{k+1}^{i}-p_{k}^{i}}{\Delta t}, \quad \frac{\partial^{2} p(r, t)}{\partial r^{2}}=\frac{\frac{p_{k}^{i+1}-p_{k}^{i}}{r_{k}^{i+1}-r_{k}^{i}}-\frac{p_{k}^{i}-p_{k}^{i-1}}{r_{k}^{i}-r_{k}^{i-1}}}{\frac{1}{2}\left(r_{k}^{i+1}-r_{k}^{i-1}\right)}
$$

where $\Delta t$ is the sampling time. The superscript $i$ and subscript $k$ in $p_{k}^{i}$ denote the value of the distributed phenomenon at the discretization node $i$ and at the time step $k$. Plugging the finite differences (2) into the mathematical model of the distributed phenomenon (1), in general, leads to a system of polynomial equations of degree three. However, for the case of one unknown sensor node location, this reduces to a single quadratic equation, as shown in the next subsection.

2) Solving Polynomial System Equations: Based on the spatial and temporal discretization, the partial differential equation (1) may be expressed as a finite difference equation and put in the following form at each discretization point, $p_{k}^{i}$, in the interval in question

$$
\begin{aligned}
0= & A_{k}^{i}\left(r_{k}^{i+1}-r_{k}^{i}\right)\left(r_{k}^{i}-r_{k}^{i-1}\right)\left(r_{k}^{i+1}-r_{k}^{i-1}\right) \\
& -B_{k}^{i}\left(r_{k}^{i}-r_{k}^{i-1}\right)+C_{k}^{i}\left(r_{k}^{i+1}-r_{k}^{i}\right)
\end{aligned}
$$

where

$A_{k}^{i}=\frac{p_{k+1}^{i}-p_{k}^{i}}{2 \alpha \Delta t}, \quad B_{k}^{i}=p_{k}^{i+1}-p_{k}^{i}, \quad C_{k}^{i}=p_{k}^{i}-p_{k}^{i-1}$.

At this point, it is important to mention that $r_{k}^{i}$ represents the unknown location of the sensor node to be localized and $r_{k}^{i+1}$ and $r_{k}^{i-1}$ are the known locations of neighboring nodes, visualized in Fig. 2 (b). The derived system equation (3) can be simply regarded as an explicit relation between three positions on a line (two known endpoints and one unknown location between them), and four values of the measured phenomenon (all known and one at each location at time $t$ and one at the unknown location at time $t+1$ ). In order to derive the unknown location $r_{k}^{i}$ of sensor node $i$, the polynomial system of equations (3) needs to be solved and the root selected, which best fits the conditions (e.g., must be between the two known locations $r_{k}^{i-1}$ and $r_{k}^{i+1}$ ).
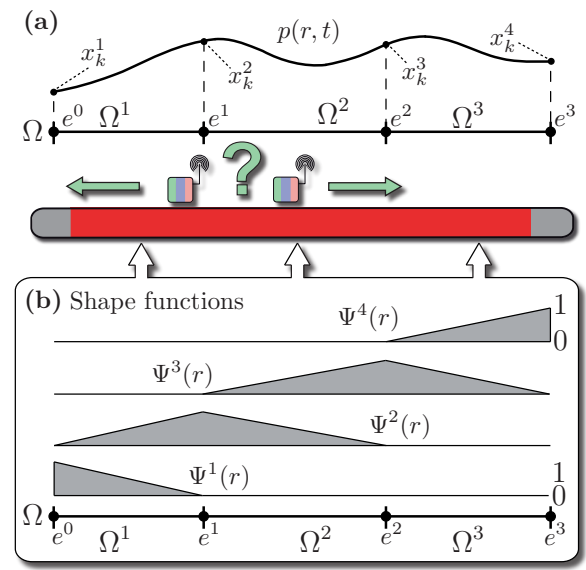

Fig. 3. The solution $\boldsymbol{p}(r, t)$ of the distributed phenomenon is approximated by a series of shape functions $\Psi_{i}(r)$ and their respective weighting coefficients $\boldsymbol{x}_{k}^{i}$. (a) Elemental decomposition of solution domain $\Omega$ into several subdomains $\Omega^{e}$ and (b) application of shape functions $\Psi_{k}(r)$ [2].

The PSL-method assumes a densely deployed sensor network in which every node $i$ communicates with its neighboring nodes $i-1$ and $i+1$. This means that measurements of the distributed phenomenon $p_{k}^{i-1}$ and $p_{k}^{i+1}$ need to be transmitted between adjacent nodes, see Fig. 2 (b). It can be stated that the denser the sensor nodes are deployed, the more accurate the individual nodes in the network can be localized. The proposed localization approach involves neither errors in the mathematical model nor uncertainties in the measurements. However, it can be easily implemented and has low computational complexity.

\section{Finite-Dimensional Model FOR NODE LOCALIZATION}

In this section, we derive a finite-dimensional model, which can be exploited for the simultaneous reconstruction of distributed phenomena and node localization based on local measurements. The finite-dimensional model consists of two parts: the system model and the measurement model. The system model describes the dynamic behavior of the distributed phenomenon to be monitored in terms of a finite-dimensional state vector $\underline{\boldsymbol{x}}_{k}$. On the other hand, the measurement model relates the actual measurements obtained by the sensor network both to the state vector $\underline{x}_{k}$ describing the phenomenon and to the current node locations $\boldsymbol{\eta}_{k}^{S}$.

\section{A. Conversion of Distributed Phenomena}

The model-based state estimation of distributed systems based on a distributed-parameter description (1) is quite complex. The reason is that a Bayesian estimation method usually exploits a lumped-parameter system description. In order to cope with this problem, the system description has to be converted from a distributed-parameter form into a lumped-parameter form. In general, the conversion of the system description (1) can be achieved by methods for solving partial differential equations, such as modal analysis [4], the finite-difference method [7], [8], the finite-element method [2], and finite-spectral method [13]. Basically, these 
methods consist of two steps, namely spatial decomposition and temporal decomposition.

1) Spatial decomposition: By means of the spatial decomposition, the partial differential equation (1) is converted into a set of ordinary differential equations [2]. First, the solution domain $\Omega=\{r \mid 0 \leq r \leq L\}$ needs to be decomposed into $N$ subdomains $\Omega^{e}$. Then, the solution $\boldsymbol{p}(r, t)$ in the entire domain $\Omega$ is represented by a piecewise approximation according to

$$
\boldsymbol{p}(r, t)=\sum_{i=1}^{N} \Psi^{i}(r) \boldsymbol{x}^{i}(t)
$$

where $\Psi^{i}(r)$ are analytic functions called shape functions. It is important to note that the individual shape functions $\Psi^{i}(r)$ are defined on the entire solution domain. The essence of all aforementioned conversion methods lies in the choice of the shape functions $\Psi^{i}(r)$, e.g., piecewise linear functions, orthogonal functions, or trigonometric functions [2].

2) Temporal discretization: In order to derive a discretetime system model the system of ordinary differential equations (derived from the spatial decomposition) needs to be discretized in time. The temporal discretization produces a linear system of equations for the state vector $\underline{\boldsymbol{x}}_{k}$ containing the unknown weighting factors of the finite expansion (4). The resulting discrete-time lumped-parameter system represents the approximation of the distributed system (1).

In the case of linear partial differential equations (1), the aforementioned methods for the spatial and temporal decomposition always result in a linear system of equations according to

$$
\underline{\boldsymbol{x}}_{k+1}=\mathbf{A}_{k} \underline{\boldsymbol{x}}_{k}+\mathbf{B}_{k}\left(\underline{\hat{\hat{x}}}_{k}+\underline{\boldsymbol{w}}_{k}^{x}\right) \text {. }
$$

The global state vector $\underline{x}_{k}$ characterizes the state of the distributed system and the vector $\underline{\boldsymbol{w}}_{k}^{x}$ represents the system uncertainties. The structure of the system matrix $\mathbf{A}_{k}$ and the input matrix $\mathbf{B}_{k}$ merely depends on the applied conversion method [2].

\section{B. Measurement Model for Node Localization}

In this section, we derive the measurement model for the purpose of localizing sensor nodes based on local observations of a physical phenomenon. The measurement model consists of two parts, namely the measurement equation and the output equation, described in the following.

1) Measurement equation: The measurement equation relates the actual measurements $\hat{y}_{k}^{i}$ at location $\boldsymbol{\eta}_{k}^{i}$ to the distributed phenomenon $\boldsymbol{p}\left(r, t_{k}\right)$, according to

$$
\underline{\hat{y}}_{k}=\underline{h}_{k}^{*}\left(\boldsymbol{p}\left(r, t_{k}\right)\right)+\underline{\boldsymbol{v}}_{k}^{*},
$$

where the vector $\underline{\boldsymbol{v}}_{k}^{*}$ contains the uncertainties arising from the actual sensor node. In general, depending on the measurement principle used for the actual sensor, the mapping $\underline{h}_{k}^{*}(\cdot)$ consists of nonlinear functions.
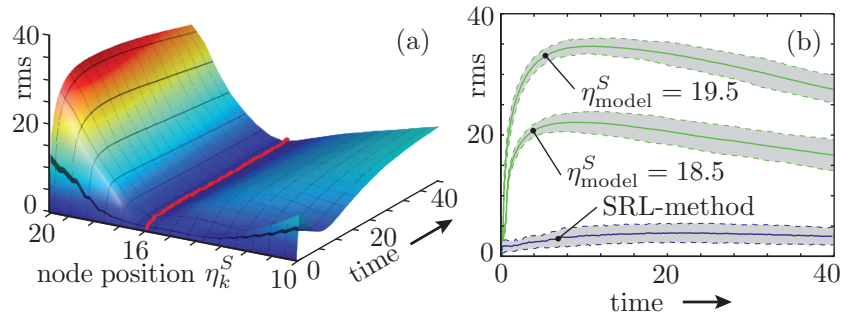

Fig. 4. Visualization of root mean square error (rms) and error variance averaged over 100 Monte Carlo simulation runs. The true node location is assumed to be $\eta_{\text {true }}^{S}=16$. (a) Rms of Kalman filter based on incorrect node locations. It is obvious that with the deviation of the node location the performance quickly degrades. (b) Comparison of Kalman filter (green) based on incorrect node locations $\eta_{\text {model }}^{S}=[18.5,19.5]$ and the simultaneous reconstruction and node localization (SRL-method) approach (blue).

2) Output equation: The output equation relates the finitedimensional state vector $\underline{\boldsymbol{x}}_{k}$ directly to the distributed phenomenon $\boldsymbol{p}\left(r, t_{k}\right)$ in continuous space, according to

$$
\boldsymbol{p}\left(r, t_{k}\right)=\sum_{j=1}^{N} \Psi^{j}(r) \boldsymbol{x}_{k}^{j},
$$

where $\Psi^{j}(r)$ represents the shape functions. It is important to emphasize that the shape functions $\Psi_{j}(r)$ here are identical to the shape functions used for the spatial decomposition.

In this article, we assume that the sensor nodes measure directly a realization of the distributed phenomenon $\boldsymbol{p}\left(\boldsymbol{\eta}_{k}^{i}, t_{k}\right)$ at their individual locations $\boldsymbol{\eta}_{k}^{i}$. Then, the measurement equation for the entire network is assembled from the individual shape functions $\Psi^{j}\left(\boldsymbol{\eta}_{k}^{i}\right)$ according to

$$
\underline{\hat{y}}_{k}=\underbrace{\left[\begin{array}{ccc}
\Psi^{1}\left(\boldsymbol{\eta}_{k}^{1}\right) & \cdots & \Psi^{N}\left(\boldsymbol{\eta}_{k}^{1}\right) \\
\vdots & \ddots & \vdots \\
\Psi^{1}\left(\boldsymbol{\eta}_{k}^{M}\right) & \cdots & \Psi^{N}\left(\boldsymbol{\eta}_{k}^{M}\right)
\end{array}\right]}_{\mathbf{H}_{k}\left(\underline{\boldsymbol{\eta}}_{k}^{S}\right)} \underline{\boldsymbol{x}}_{k}+\underline{\boldsymbol{v}}_{k},
$$

where $\underline{\boldsymbol{v}}_{k}$ denotes the measurement uncertainty and $M$ represents the number of sensor nodes used in the network. The measurement model (6) directly relates the measurements $\hat{y}_{k}^{i}$ to the state vector $\underline{\boldsymbol{x}}_{k}$ characterizing the distributed phenomenon and to the location vector $\boldsymbol{\eta}_{k}^{S}$ containing the individual node locations $\underline{\boldsymbol{\eta}}_{k}^{S}=\left[\boldsymbol{\eta}_{k}^{1}, \ldots, \overline{\boldsymbol{\eta}}_{k}^{M}\right]$. The structure of the measurement matrix $\mathbf{H}_{k}$ for localizing sensor nodes in a network is shown in the following example.

\section{Example 1 (Measurement model for node localization)} In this example, we visualize the structure of the measurement matrix $\mathbf{H}_{k}$ subject to piecewise linear shape functions. The entire solution domain $\Omega$ is decomposed into 3 subdomains and appropriate piecewise linear functions are defined on each subdomain. In addition, there are two sensor nodes located at $\boldsymbol{\eta}_{k}^{1}$ and $\boldsymbol{\eta}_{k}^{2}$ in the subdomains $\Omega^{1}$ and $\Omega^{2}$, as depicted in Fig. 3 . Then, the measurement model is given as follows

$$
\left[\begin{array}{c}
\hat{y}_{k}^{1} \\
\hat{y}_{k}^{2}
\end{array}\right]=\left[\begin{array}{cccc}
\overbrace{c_{1}^{1}+c_{2}^{1}\left(\boldsymbol{\eta}_{k}^{1}\right)}^{\overbrace{k}^{1})} & \overbrace{c_{3}^{1}+c_{4}^{1} \boldsymbol{\eta}_{k}^{1}}^{\left.\Psi_{k}^{1} \boldsymbol{\eta}_{k}^{1}\right)} & 0 & 0 \\
0 & \underbrace{c_{1}^{2}+c_{2}^{2} \boldsymbol{\eta}_{k}^{2}}_{\Psi^{2}\left(\boldsymbol{\eta}_{k}^{2}\right)} & \underbrace{c_{3}^{2}+c_{4}^{2} \boldsymbol{\eta}_{k}^{2}}_{\Psi^{3}\left(\boldsymbol{\eta}_{k}^{2}\right)} & 0
\end{array}\right]\left[\begin{array}{l}
\boldsymbol{x}_{k}^{1} \\
\boldsymbol{x}_{k}^{2} \\
\boldsymbol{x}_{k}^{3} \\
\boldsymbol{x}_{k}^{4}
\end{array}\right]+\underline{\boldsymbol{v}}_{k},
$$


where the constants $c_{i}^{j}$ arise from the definition of the piecewise linear shape functions in each subdomain and thus the geometry of the applied grid for the finite elements. The extension to orthogonal polynomials and trigonometric functions can be derived in a straightforward fashion [2], [6].

From the previous example, it is obvious that the structure of the measurement matrix $\mathbf{H}_{k}$ merely depends on the location $\underline{\boldsymbol{\eta}}_{k}^{S}$ of the individual sensor nodes. That means, for the accurate reconstruction of the distributed phenomenon (1) based on a sensor network, the exact node locations $\underline{\boldsymbol{\eta}}_{k}^{S}$ are necessary. Due to this dependency, deviations of true locations from the modeled node locations lead to poor estimation results, as shown in Sec. V-A.

On the other hand, thanks to the dependency of the measurement matrix $\mathbf{H}_{k}$ on the node locations $\underline{\boldsymbol{\eta}}_{k}^{S}$, the localization problem can be stated as a simultaneous state and parameter estimation problem. By this means, the distributed phenomenon can be reconstructed and the sensor nodes can be localized in a simultaneous fashion. In the next section, we introduce the method for the simultaneous reconstruction and node localization (SRL-method).

\section{Simultaneous Reconstruction of Distributed PHENOMENA AND NODE LOCALIZATION}

After the derivation of a finite-dimensional model for the node localization in the previous section, we now introduce a method for the simultaneous reconstruction of distributed phenomena and node localization (SRL-method). There are four key features characterizing the novelties of the proposed method: (a) approach is based on local measurements only, (b) systematic consideration of uncertainties in the model description and the measurements, (c) derivation of an uncertainty measure for the estimated node location in terms of a density function, and (d) the simultaneous approach allows improving the estimation of the distributed phenomenon.

\section{A. Reconstruction based on Incorrect Node Locations}

For the reconstruction of distributed phenomena, an appropriate estimator has to be developed. The structure of the estimator usually depends on the system model and the measurement model, i.e., being linear or nonlinear. For the pure reconstruction of the state $\boldsymbol{p}(r, t)$ of linear distributed phenomena (1), both system equation (5) and measurement equation (6) are linear. Therefore, it is sufficient to use the Kalman filter for the reconstruction of the distributed phenomenon $\boldsymbol{p}(r, t)$.

The Kalman filter requires a rather precise model of the system and a precisely known uncertainty description. If any of these assumptions is violated, the performance of the reconstruction process can quickly degrade. In many cases, the locations of sensor nodes (randomly deployed or movable nodes) contain some uncertainties or even could be completely unknown. The degradation leading to poor performance is illustrated in the next example.

Example 2 (Reconstruction with incorrect node location) In this example, we consider the one-dimensional diffusion equation (1) subject to Dirichlet boundary condition at both

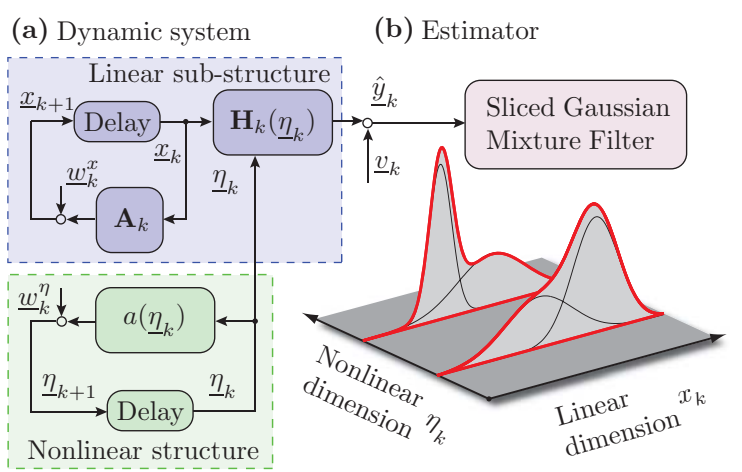

Fig. 5. Visualization of dynamic system and estimator for the node localization based on local observations. (a) The system description contains a high-dimensional linear substructure. The node location $\underline{\boldsymbol{\eta}}_{k}$ characterizes the measurement matrix $\mathbf{H}_{k}$, and thus the individual measurements. (b) The estimator is based on sliced Gaussian mixture densities consisting of a Gaussian mixture in $\boldsymbol{x}_{k}$ subspace and Dirac mixture in $\boldsymbol{\eta}_{k}$ subspace. By this means, the localization problem can be decomposed into nonlinear and linear problems.

ends and respective initial conditions, as depicted in Fig. 2 (a). The nominal parameters for the system model (1) are given by

$$
s(r, t)=0, \quad \alpha(r, t)=1, \quad \Delta t=0.2, \quad \eta_{\text {true }}^{S}=16,
$$

where $\eta_{\text {true }}^{S}$ denotes the true node location. The system uncertainty at the individual discretization nodes is given by $C_{k}^{w i}=$ 20 and the measurement noise variance by $C_{k}^{v}=0.5$. The reconstruction of the distributed phenomenon is performed on the basis of a Kalman filter with nominal parameter set for the sensor location $\eta_{\text {model }}^{S}$ according to

$$
\eta_{\text {model }}^{S}=\left[\begin{array}{lllll}
10, & 10.5, & \ldots, & 19.5, & 20
\end{array}\right] .
$$

For each assumed node location, 100 independent Monte Carlo simulation runs have been performed, resulting in $n=100$ true realizations $\underline{\widetilde{x}}_{k}^{i}$ of the distributed phenomenon.

The simulation results are shown in Fig. 4. The root mean square error (rms) and the error variance are approximated by calculating the average according to

$\hat{e}_{k}^{2} \approx \frac{1}{n \cdot m} \sum_{i=1}^{n}\left\|\underline{x}_{k}^{i}-\underline{\hat{x}}_{k}^{i}\right\|, \quad C_{k}^{\mathrm{rms}} \approx \frac{1}{n-1} \sum_{i=1}^{n}\left(e_{k}^{i}-\hat{e}_{k}\right)^{2}$,

where $\underline{\hat{x}}_{k}^{i}$ denotes the mean of the estimated state vector. The root mean square error $\hat{e}_{k}$ of the Kalman filter based on incorrect node locations is shown in Fig. 4 (a). It is obvious that the more the assumed node location deviates from the true location, the more the performance of the reconstruction result degrades. Fig. 4 (b) depicts the comparison of the estimation error between the Kalman filter based on incorrect node locations and the simultaneous node localization and reconstruction approach (SRL-method); see Sec. V-B. Thanks to the simultaneous approach, the estimation of the distributed phenomenon can be significantly improved.

\section{B. Augmented System Description for the Node Localization}

For the simultaneous node localization and reconstruction of distributed phenomena, the unknown locations of the sensor nodes $\underline{\boldsymbol{\eta}}_{k}^{S}$ are treated as additional state variables. By this means, conventional estimation techniques can be used to simultaneously estimate the location and the state 
of the distributed phenomenon. Hence, an augmented state vector $\underline{\boldsymbol{z}}_{k}$ containing the system state $\underline{\boldsymbol{x}}_{k}$ and the additional unknown node locations $\underline{\boldsymbol{\eta}}_{k}^{S}$ is defined by $\underline{\boldsymbol{z}}_{k}:=\left[\underline{\boldsymbol{x}}_{k}^{T}, \underline{\boldsymbol{\eta}}_{k}^{T}\right]^{T}$.

The augmentation of the state vector with additional unknown parameters leads to the so-called augmented system model. In the case of localizing sensor nodes, the augmentation leads to following augmented system model

$$
\left[\begin{array}{c}
\underline{\boldsymbol{x}}_{k+1} \\
\underline{\boldsymbol{\eta}}_{k+1}^{S}
\end{array}\right]=\left[\begin{array}{c}
\mathbf{A}_{k} \underline{\boldsymbol{x}}_{k}+\mathbf{B}_{k} \hat{\hat{u}}_{k} \\
\underline{a}_{k}\left(\underline{\boldsymbol{\eta}}_{k}^{S}\right)
\end{array}\right]+\left[\begin{array}{c}
\mathbf{B}_{k} \underline{\boldsymbol{w}}_{k}^{x} \\
\underline{\boldsymbol{w}}_{k}^{S}
\end{array}\right],
$$

and measurement model

$$
\underline{\hat{y}}_{k}=\underbrace{\mathbf{H}_{k}\left(\underline{\boldsymbol{\eta}}_{k}^{S}\right) \underline{\boldsymbol{x}}_{k}}_{\underline{h}_{k}\left(\underline{\boldsymbol{x}}_{k}, \underline{\boldsymbol{\eta}}_{k}^{S}\right)}+\underline{\boldsymbol{v}}_{k},
$$

where the nonlinear function $\underline{a}_{k}(\cdot)$ describes the dynamic behavior of the node locations $\underline{\boldsymbol{\eta}}_{k}^{S}$ to be estimated.

The structure of the augmented system model (7) and (8) for the node localization is depicted in Fig. 5 (a). In this case, it is obvious that the augmented measurement model is nonlinear in the augmented state vector $\underline{\boldsymbol{z}}_{k}$ due to the multiplication of $\mathbf{H}_{k}\left(\underline{\boldsymbol{\eta}}_{k}^{S}\right)$ and the system state $\underline{\boldsymbol{x}}_{k}$, see Example 1. That means, the node locations $\underline{\boldsymbol{\eta}}_{k}^{S}$ characterize the measurement matrix $\mathbf{H}_{k}$ and thus the actual measured values. It is important to emphasize that the measurement model (8) contains a high-dimensional linear substructure, which can be exploited by the application of a more efficient estimator. In the following section, we describe a novel estimator - the Sliced Gaussian Mixture Filter - allowing the decomposition of the localization problem.

\section{Estimation based on Sliced Gaussian Mixture Densities}

There are several methods to exploit the linear substructure in the combined linear/nonlinear system equation (7) and measurement equation (8). The marginalized particle filter [14] integrates over the linear subspace in order to reduce the dimensionality of the state-space. Based on this marginalization, the standard particle filter is extended by applying the Kalman filter to find the optimal estimate for the linear subspace (which is associated with the respective individual particles). The marginalized filter certainly improves the performance in comparison with the standard particle filter. However, some drawbacks still remain. For instance, special measures have to be taken in order to avoid effects like sample degeneration and impoverishment. More importantly, it does not provide a measure on how well the true joint density is represented by the estimated one.

For that reason, a more systematic estimator is employed for the simultaneous reconstruction of distributed system and node localization. This localization method is based on a special kind of density allowing a decomposition of the estimation problem. To be more specific, as a density representation we employ a so-called sliced Gaussian mixture density and a systematic approximation method leading to (close to) optimal estimation results, see Fig. 5 (b).

The sliced Gaussian mixture density $f\left(\underline{x}_{k}, \underline{\eta}_{k}^{S}\right)$ is represented by a Dirac mixture in the nonlinear subspace

$$
\begin{array}{|c|}
\hline \multicolumn{1}{|c|}{\text { Conditional linear subspace }} \\
\hline \hline \gamma_{k}^{i j}:=\mathcal{N}\left(\hat{\underline{y}}_{k}-\mathbf{H}_{k}^{i} \underline{\mu}_{k}^{p}, \mathbf{H}_{k}^{i} \mathbf{C}_{k}^{p i j} \mathbf{H}_{k}^{i T}+\mathbf{C}_{v}\right) \\
\underline{\mu}_{k}^{e i j}:=\underline{\mu}_{k}^{p i j}+\mathbf{K}\left(\underline{\hat{y}}_{k}-\mathbf{H}_{k}^{i} \underline{\mu}_{k}^{p i j}\right) \\
\mathbf{C}_{k}^{\text {eij }}:=\mathbf{C}_{k}^{p i j}-\mathbf{K H}_{k}^{i} \mathbf{C}_{k}^{p i j} \\
\text { with } \mathbf{K}:=\mathbf{C}_{k}^{p i j} \mathbf{H}_{k}^{i T}\left(\mathbf{C}_{v}+\mathbf{H}_{k}^{i} \mathbf{C}_{k}^{p i j} \mathbf{H}_{k}^{i T}\right)^{-1} \\
\hline
\end{array}
$$

TABLE I

Filter SteP: Parameters of estimated Density.

\begin{tabular}{|l|l|}
\hline Nonlinear subspace & \multicolumn{1}{|c|}{ Conditional linear subspace } \\
\hline \hline$\underline{\xi}_{k+1}^{p i}:=\underline{a}_{k}\left(\underline{\xi}_{k}^{e i}\right)$ & $\underline{\mu}_{k+1}^{p i j}:=\mathbf{A}_{k} \underline{\mu}_{k}^{e i j}+\mathbf{B}_{k} \underline{\hat{u}}_{k}$ \\
$\mathbf{C}_{w}^{n}$ & $\mathbf{C}_{k+1}^{p i j}:=\mathbf{A}_{k} \mathbf{C}_{k}^{e i j} \mathbf{A}_{k}^{T}+\mathbf{C}_{w}^{l}$ \\
\hline
\end{tabular}

TABLE II

Prediction SteP: PARAMETERS OF PREDicted DENSity.

$\underline{\boldsymbol{\eta}}_{k}^{S}$ (node locations) and a Gaussian mixture in the linear subspace $\underline{\boldsymbol{x}}_{k}$ (state vector),

$$
f\left(\underline{x}_{k}, \underline{\eta}_{k}^{S}\right)=\sum_{i=1}^{M} \alpha_{k}^{i} \underbrace{\delta\left(\underline{\eta}_{k}^{S}-\underline{\xi}_{k}^{i}\right)}_{\text {Dirac mixture }} \underbrace{f\left(\underline{x}_{k} \mid \underline{\xi}_{k}^{i}\right)}_{\text {Gaussian mixture }} .
$$

The density parameters $\underline{\xi}_{k}^{i} \in \mathbb{R}^{s}$ can be regarded as the position of the individual components of the sliced Gaussian mixture densities $f\left(\underline{x}_{k}, \underline{\eta}_{k}^{S}\right)$ as shown in Fig. 5 (b). The marginal density in nonlinear subspace $\underline{\boldsymbol{\eta}}_{k}^{S}$ is given by a Dirac mixture function, according to

$$
f\left(\underline{\eta}_{k}^{S}\right)=\sum_{i=1}^{M} \alpha_{k}^{i} \delta\left(\underline{\eta}_{k}^{S}-\underline{\xi}_{k}^{i}\right),
$$

where $\alpha_{k}^{i}$ and $\underline{\xi}_{k}^{i}$ represent the weights and positions of the Dirac functions, respectively. The density representation along the individual slices is assumed to be a Gaussian mixture density

$$
f\left(\underline{x}_{k} \mid \underline{\xi}_{k}^{i}\right)=\sum_{j=1}^{N^{i}} \beta_{k}^{i j} \mathcal{N}\left(\underline{x}_{k}-\underline{\mu}_{k}^{i j}, \mathbf{C}_{k}^{i j}\right),
$$

with $\beta_{k}^{i j}, \underline{\mu}_{k}^{i j} \in \mathbb{R}^{r}$, and $\mathbf{C}_{k}^{i j} \in \mathbb{R}^{r \times r}$ denoting the weights, means, and covariance matrices of the $j$-th component of the Gaussian mixture density of the $i$-th slice.

Thanks to the conditionally linear dynamic system model (7) and measurement model (8), the ChapmanKolmogorov equation for the prediction step and the Bayes formula for the measurement step can be solved analytically. The proof is omitted here; rather the resulting predicted density is stated. By means of the sliced Gaussian mixture filter, the predicted density $\widetilde{f}^{p}$ results in a Gaussian mixture both in linear $\underline{\boldsymbol{x}}_{k}$ and nonlinear subspace $\underline{\boldsymbol{\eta}}_{k}^{S}$,

$$
\begin{aligned}
& \tilde{f}^{p}\left(\underline{x}_{k+1}, \underline{\eta}_{k+1}^{S}\right)=c \cdot \sum_{i=1}^{M} \sum_{j=1}^{N^{i}} \alpha_{k}^{i} \beta_{k}^{i j} \gamma_{k}^{i j} \\
& \cdot \mathcal{N}\left(\underline{\eta}_{k+1}^{S}-\underline{\xi}_{k+1}^{p i}, \mathbf{C}_{w}^{n}\right) \mathcal{N}\left(\underline{x}_{k+1}-\underline{\mu}_{k+1}^{p i j}, \mathbf{C}_{k+1}^{p i j}\right),
\end{aligned}
$$




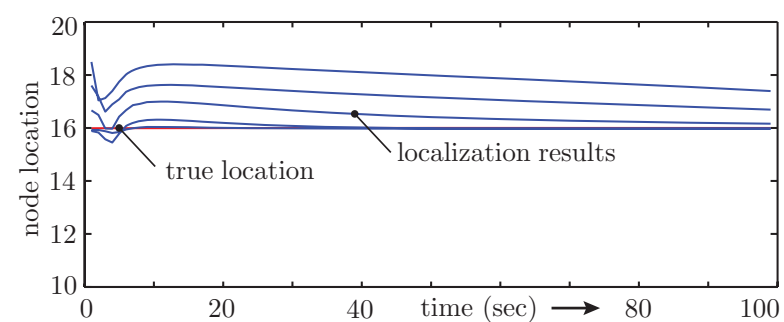

Fig. 6. Results of the polynomial systems localization method (PSLmethod) for various neighboring nodes with known locations. The true node location to be identified is $\eta_{\text {true }}^{S}=16$.

where the mean and covariance matrices in linear subspace $\underline{\boldsymbol{x}}_{k}$ are calculated by applying the standard Kalman prediction step. The mean in the nonlinear subspace $\underline{\boldsymbol{\eta}}_{k}^{S}$ is derived by simply repositioning the density slices. The parameters for the predicted density (12) are shown in Tables I and II. For the sake of simplicity and in order to keep the equations simple, the following abbreviation is used: $\mathbf{H}_{k}^{i}:=\mathbf{H}\left(\xi_{k}^{i}\right)$.

In order to bound the complexity, the predicted density (12) in terms of a Gaussian mixture density needs to be reapproximated as a sliced Gaussian mixture density (9). One possible approach for the approximation is to derive the location of the density slices by only considering the marginal density $\widetilde{f}^{p}\left(\underline{\eta}_{k+1}^{S}\right)$. In general, this approximation of arbitrary marginal densities $\tilde{f}^{p}\left(\underline{\eta}_{k+1}^{S}\right)$ by Dirac mixture densities (10) can be achieved by batch approximation [15] or sequential approximation [16].

After the approximation of the marginal density $\widetilde{f}^{p}\left(\underline{\eta}_{k+1}^{S}\right)$ in the nonlinear subspace, the Dirac approximation is extended to a sliced Gaussian mixture representation over the entire sample space. Basically, this is achieved by evaluating the Gaussian mixture density $\widetilde{f}^{p}\left(\underline{x}_{k+1}, \underline{\eta}_{k+1}^{S}\right)$ at every Dirac position, i.e., at every slice position, determined by the algorithm. This leads to a sliced Gaussian mixture density (9), which can be used for the next processing step. A more detailed description of this estimator can be found in [12].

\section{Simulation Results}

In this section, the performance of the proposed localization methods is demonstrated by means of simulation results.

\section{Example 3 (Simulated system)}

In this simulation, we consider the localization based on the one-dimensional partial differential equation (1), with assumed initial condition and Dirichlet boundary conditions as depicted in Fig. 2 (a). The aim is the localization of a sensor node with initially unknown location based on local observations only. The true node location is given by $\boldsymbol{\eta}_{\text {true }}^{S}=16$. The system noise term is $\mathbf{C}_{l}^{w}=\operatorname{diag}\{20, \ldots, 20\}$, the noise term for the node location is given by $C_{n}^{w}=0.02$, and for the local measurement of the node to be localized is assumed to be $C_{v}=0.01$. Here, we compare different approaches for the passive localization based on local measurements: (a) PSLmethod, (b) deterministic approach introduced in [8] (CSNmethod), (c) SRL-method based on sliced Gaussian mixture filter (50 slices), (d) SRL-method based on marginalized particle filter (500 particles). These approaches are compared based on 100 Monte-Carlo simulation runs.

The simulation results for the PSL-method are depicted in Fig. 6. It is important to mention that this deterministic approach was simulated with perfect information, i.e., there is noise neither in the system nor in the measurements. Furthermore, we assume that the sensor node to be localized receives information about distributed phenomenon and locations from neighboring nodes, see Fig. 2 (b). Since the diffusion equation has derivatives involving $\Delta t$ and $\Delta x$, the PSL-method is sensitive to the distance between the two adjacent known locations. Evidence of this effect is shown in Fig. 6 which plots the values found by the PSL-method for known points of varying distance from the unknown. It is obvious that the denser the nodes are deployed the more accurate the location can be identified.

The simulation results for the SRL-method with considering all the aforementioned uncertainties is shown in Fig. 7. Here, we assume the sensor network consists only of a single sensor node locally measuring the phenomenon. Furthermore, the sensor node has only very uncertain knowledge about the initial distributed phenomenon, see Fig. 7 (a).

Fig. 7 (c) depicts one specific simulation run for the estimation of the unknown node location $\boldsymbol{\eta}_{k}^{S}$. It is obvious that after a certain transition time the SRL-method based on sliced Gaussian mixture filter (with 50 slices) offers a nearly exact location estimation, while the determinstic approach CSN-method strongly deviates (due to neglecting system and measurement noises). The density function $f\left(\eta_{k}^{S}\right)$ for the estimated location $\boldsymbol{\eta}_{k}^{S}$ for a specific simulation run is depicted in Fig. 7 (e). It can be seen that with exploiting more and more measurements and information about the dynamic system, the estimation of the location changes from a multimodal to an unimodal function. This explains the higher uncertainty at the beginning of the simulation. The root mean square error (rms) of all 100 simulation runs over time is depicted in Fig. 7 (d). It is obvious that the SRLmethod based on Sliced Gaussian Mixture Filter (with 50 slices) outperforms both the deterministic approach (CSNmethod) and the approach based on marginalized particle filter (with 500 particles); mainly due to the consideration of uncertainties and the systematic and deterministic approximation of the density.

Comparing Fig. 7 (a) and (b), it is obvious that thanks to the simultaneous property of the SRL-method, not only can the sensor node be accurately localized, but also the estimation about the distributed phenomenon can be further improved. This can be exploited by other sensor nodes to localize themselves.

\section{CONLUSiOnS AND Future WORK}

In this paper, we introduce the methodology of two novel localization approaches for sensor nodes measuring locally only their surrounding. The PSL-method is a deterministic approach and is mainly based on restating the mathematical model in terms of the location. In the case of no noise in the model description and the measurement, this method leads to sufficient results for a dense sensor network. The stochastic SRL-method basically reformulates the localization problem into a simultaneous state and parameter estimation problem. This leads to a high-dimensional nonlinear estimation problem, which makes the employment of special types of estimators necessary. Here, the sliced Gaussian mixture 

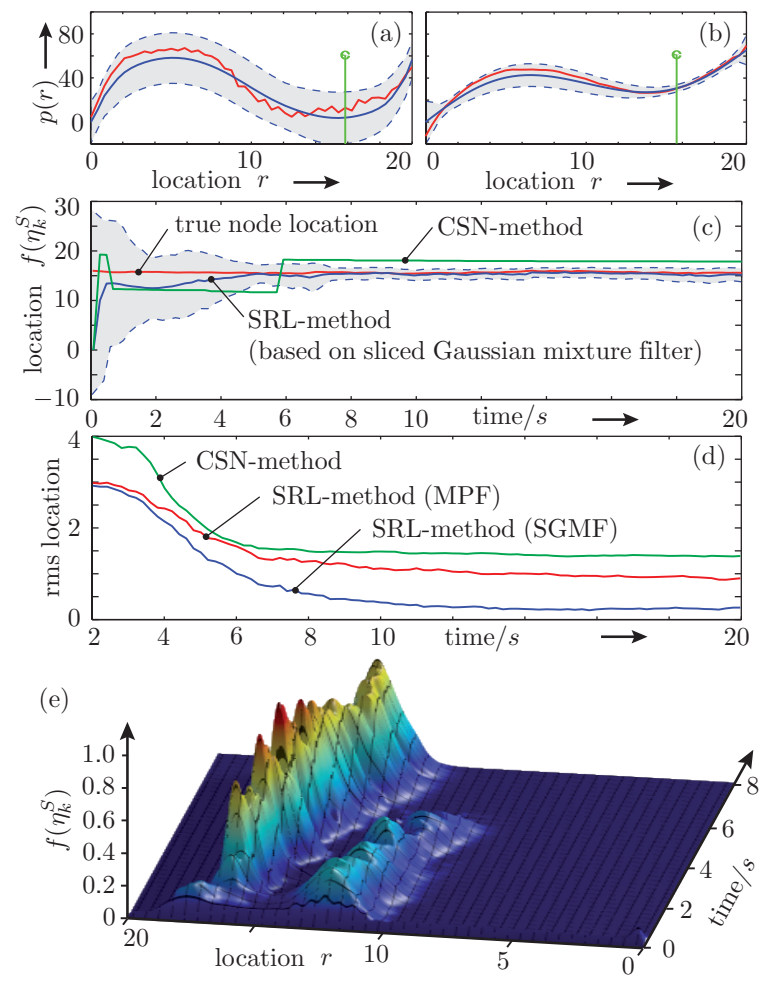

Fig. 7. Comparision of SRL-method based on SGMF (blue), SRL-method based on MPF (red), and deterministic approach CSN-method (green). (a)(b) Improvement of estimation of distributed phenomenon (blue) thanks to simultaneous approach; realization of the distributed phenomenon (red). (c) Specific simulation run for the estimation of the node location $\boldsymbol{\eta}_{k}^{S}$. The true location (red) is assumed to be $\boldsymbol{\eta}_{\text {true }}^{S}=16$. (d) Root mean square error over time of 100 simulation runs. (e) Specific density function $f\left(\eta_{k}^{S}\right)$ for estimated node location $\boldsymbol{\eta}_{k}^{S}$ over time.

filter (SGMF) and the marginalized particle filter (MPF) are applied for the decomposition of this estimation problem. Thanks to the stochastic approach, the SRL-method leads to better estimation results for the location, even with noisy information. Furthermore, the simultaneous approach allows to improve the knowledge about the phenomenon, which then can be exploited by other nodes for the localization.

The application of the proposed localization methods (PSL-method and SRL-method) to sensor networks provides novel prospects. The network is able to localize the individual nodes without relying on a satelite positioning system (which is not always available, e.g., indoor applications) as long as a strong model of the surrounding is available.

For the PSL-method it is necessary to incorporate uncertainties into the mathematical model as well as the sensors, and to study the robustness of the method in the presence of noise. Another issue for future work is that if the locations of several nodes are unknown, they may be solved separately using the method described in this paper; however, we should compare it to the simultaneous solution of the system of degree three equations. So far, the model parameters and structure were assumed to be precisely known for the SRL-method. In many real world applications, the parameters contain uncertainties. The combination of the parameter identification of distributed phenomena and the node localization is left for future work. Finally, we intend to test the proposed localization methods on real sensor data.

\section{ACKNOWLEDGMENTS}

This work was partially supported by the German Research Foundation (DFG) within the Research Training Group GRK 1194 "Self-organizing Sensor-ActuatorNetworks".

\section{REFERENCES}

[1] D. Culler, D. Estrin, and M. Srivastava, "Overview of Sensor Networks," IEEE Computer, vol. 37, no. 8, pp. 41-49, 2004.

[2] F. Sawo, K. Roberts, and U. D. Hanebeck, "Bayesian Estimation of Distributed Phenomena using Discretized Representations of Partial Differential Equations," in Proceedings of the 3rd International Conference on Informatics in Control, Automation and Robotics (ICINCO 2006), Setúbal, Portugal, Aug. 2006, pp. 16-23.

[3] T. Zhao and A. Nehorai, "Detecting and Estimating Biochemical Dispersion of a Moving Source in a Semi-Infinite Medium," IEEE Transactions on Signal Processing, vol. 54, no. 6, pp. 2213-2225, June 2006.

[4] T. Bader, A. Wiedemann, K. Roberts, and U. D. Hanebeck, "Modelbased Motion Estimation of Elastic Surfaces for Minimally Invasive Cardiac Surgery," in Proceedings of the 2007 IEEE International Conference on Robotics and Automation (ICRA 2007), Rome, Italy, Apr. 2007, pp. 2261-2266.

[5] A. Jeremic and A. Nehorai, "Design of Chemical Sensor Arrays for Monitoring Disposal Sites on the Ocean Floor," IEEE Journal of Oceanic Engineering, vol. 23, pp. 334-343, 1998.

[6] F. Sawo, V. Klumpp, and U. D. Hanebeck, "Simultaneous State and Parameter Estimation of Finite-Dimensional Models of Distributed Systems based on Sliced Gaussian Mixture Filter," in Proceedings of the 11th International Conference on Information Fusion (Fusion 2008), Cologne, Germany, Jul. 2008.

[7] L. A. Rossi, B. Krishnamachari, and C.-C. Kuo, "Distributed Parameter Estimation for Monitoring Diffusion Phenomena Using Physical Models," in First Annual IEEE Communications Society Conference on Sensor and Ad Hoc Communications and Networks (SECON 2006), Los Angeles, USA, 2004, pp. 460-469.

[8] T. C. Henderson, C. Sikorski, E. Grant, and K. Luthy, "Computational Sensor Networks," in Proceedings of the 2007 IEEE/RSJ International Conference on Intelligent Robots and Systems (IROS 2007), San Diego, USA, 2007.

[9] J. Hightower and G. Borriello, "Location Systems for Ubiquitous Computing," IEEE Computer, vol. 34, no. 8, pp. 57-66, August 2001.

[10] M. Grigoras, O. Feiermann, and U. D. Hanebeck, "DataDriven Modeling of Signal Strength Distributions for Localization in Cellular Radio Networks (Datengetriebene Modellierung von Feldstärkeverteilungen für die Ortung in zellulären Funknetzen)," at - Automatisierungstechnik Automatisierungstechnik, Sonderheft: Datenfusion in der Automatisierungstechnik, vol. 53, no. 7, pp. 314321, Jul. 2005.

[11] H. Wang, H. Lenz, A. Szabo, and U. D. Hanebeck, "Fusion of Barometric Sensors, wlan Signals and Building Information for 3d Indoor/Campus Localization," in Proceedings of the 2006 IEEE International Conference on Multisensor Fusion and Integration for Intelligent Systems (MFI 2006), Heidelberg, Germany, Sep. 2006.

[12] V. Klumpp, F. Sawo, U. D. Hanebeck, and D. Fränken, "The Sliced Gaussian Mixture Filter for Efficient Nonlinear Estimation," in 11th International Conference on Information Fusion (Fusion 2008), Cologne, Germany, 2008.

[13] G. E. Karniadakis and S. Sherwin, Spectral/hp Element Methods for Computational Fluid Dynamics. Oxford University Press, 2005.

[14] T. Schön, F. Gustafsson, and P.-J. Nordlund, "Marginalized Particle Filters for Nonlinear State-space Models,” Linköping University, Tech. Rep., 2003.

[15] O. C. Schrempf and U. D. Hanebeck, "A State Estimator for Nonlinear Stochastic Systems Based on Dirac Mixture Approximations," in Proceedings of the 4th International Conference on Informatics in Control, Automation and Robotics (ICINCO 2007), Angers, France, May 2007, pp. 54-61.

[16] U. D. Hanebeck and O. C. Schrempf, "Greedy Algorithms for Dirac Mixture Approximation of Arbitrary Probability Density Functions," in Proceedings of the 2007 IEEE Conference on Decision and Control (CDC 2007), New Orleans, Louisiana, Dec. 2007, pp. 3065-3071. 